\title{
Controle de ferrugem na cultura do alho com uma nova mistura de fungicidas
}

\author{
Maristella Dalla Pria ${ }^{1}$; Jeferson Zagonel${ }^{1}$, Eliana C Fernandes ${ }^{2}$ \\ ${ }^{1}$ UEPG, Dep ${ }^{\text {to }}$ Fitotecnia e Fitossanidade, Av. Carlos Cavalcanti, 4748, 84030-900 Ponta Grossa-PR; ${ }^{2}$ UEPG, mestranda em agricultura; \\ jefersonzagonel@uol.com.br; mdallapria@uol.com.br; elianacfernandes@uol.com.br
}

\begin{abstract}
RESUMO
Com o objetivo de verificar a vantagem da adição do fungicida trifloxystrobin ao tebuconazole no controle de ferrugem (Puccinia porri) na cultura do alho, foi instalado um experimento na Fazenda Escola "Capão da Onça" da UEPG, em Ponta Grossa, PR, no ano de 2003. O delineamento experimental utilizado foi de blocos ao acaso com seis tratamentos e quatro repetições. Os tratamentos constaram de tebuconazole na dose de $200 \mathrm{~g} \mathrm{ha}^{-1}\left(1,00 \mathrm{~L} \mathrm{ha}^{-1}\right.$ de Folicur 200 $\mathrm{CE}$ ); e das misturas formuladas (prontas) de trifloxystrobin e tebuconazole nas doses de 50 e 100 e 75 e $150 \mathrm{~g} \mathrm{ha}^{-1}(0,50$ e $0,75 \mathrm{~L}$ ha $^{-1}$ de Nativo SC); trifloxystrobin e tebuconazole nas doses 50 e 100 e $75 \& 150 \mathrm{~g} \mathrm{ha}^{-1}\left(0,20\right.$ e $0,30 \mathrm{~kg} \mathrm{ha}^{-1}$ de Nativo WG) e testemunha. Foram realizadas três aplicações dos tratamentos, aos 85, 96 e 103 dias após a emergência da cultura, cultivar Caçador. As avaliações de controle foram realizadas aos 11, 18 e 27 dias após a primeira aplicação dos fungicidas. Nas condições em que foi desenvolvido o experimento, de alta severidade da doença, pode-se concluir que a mistura de trifloxystrobin e tebuconazole na dose de 75 e $150 \mathrm{~g} \mathrm{ha}^{-1}$ das formulações SC e WG é adequada ao controle da ferrugem do alho pela similaridade de controle ao tebuconazole que é recomendado para o controle da doença e por não causar danos visíveis na cultura.
\end{abstract}

Palavras-chave: Allium sativum L., Puccinia porri, tebuconazole, trifloxystrobin.

\section{ABSTRACT \\ Rust control in the garlic culture with a new mixture of fungicides}

An experiment was carried out in Ponta Grossa, Paraná State, Brazil, to evaluate the advantage of the addition of the fungicide trifloxystrobin to tebuconazole, to control the garlic rust (Puccinia porri). The experimental design was of complete randomized blocks with six treatments and four replications. The treatments consisted of tebuconazol in the rate of $200 \mathrm{~g} \mathrm{ha}^{-1}\left(1,00 \mathrm{~L} \mathrm{ha}^{-1}\right.$ of Folicur $\left.200 \mathrm{CE}\right)$; and of the formulated mixtures of trifloxystrobin and tebuconazol in the rates of 50 and 100 e 75 and $150 \mathrm{~g} \mathrm{ha}^{-1}\left(0.50\right.$ and $0.75 \mathrm{~L} \mathrm{ha}^{-1}$ of Nativo SC); trifloxystrobin and tebuconazol in the rates of 50 and 100 e 75 and $150 \mathrm{~g} \mathrm{ha}^{-1}\left(0.20\right.$ and $0.30 \mathrm{~kg} \mathrm{ha}^{-1}$ of Nativo WG) and non sprayed control. Three applications of the fungicides were carried out at 85, 96, and 103 days after the plant emergence, cultivar Caçador. Disease was assessed at 11, 18 and 27 days after the first application of fungicides. Under Puccinia porri high severity observed in the experiment, the mixture of the trifloxystrobin and tebuconazol in the rate of 75 and $150 \mathrm{~g} \mathrm{ha}^{-1}$ of SC and WG, showed similar control of the garlic rust as compared to tebuconazol, fungicide recommended for the rust control and for not causing visible damages in the culture.

Keywords: Allium sativum L., Puccinia porri, tebuconazol, trifloxystrobin.

\section{(Recebido para publicação em 9 de janeiro de 2007; aceito em 18 de abril de 2008)}

A ferrugem é uma doença de ocorrência generalizada em todas as regiões do globo desde que, durante o cultivo das plantas suscetíveis, ocorram condições ambientais favoráveis ao seu desenvolvimento. A doença ocorre mais freqüentemente em condições de alta umidade relativa do ar e baixo índice pluviométrico. Temperaturas moderadas favorecem a infecção, sendo a mesma inibida quando valores acima de $24^{\circ} \mathrm{C}$ e abaixo de $10^{\circ} \mathrm{C}$ são registrados (Massola Jr. et al., 2005). Apesar de incidir sobre várias espécies do gênero Allium, a doença é especialmente importante para o alho (Allium sativum L.) e para a cebolinha (Allium fistulosum L.), sendo menos freqüente e apresentando importância relativa às demais plantas daquele gênero. Nas condições do Sul do Brasil, se situa entre as doenças de maior importância, sendo muitas vezes responsável por queda acentuada da produção de culturas nas quais não são adotadas as medidas de controle necessárias (Sanchez et al., 2003; Massola Jr. et al., 2005).

As plantas de alho são suscetíveis à ocorrência da ferrugem em qualquer fase do seu desenvolvimento. A doença, causada por Puccinia porri (D.C.) Rud. é caracteriza pelo aparecimento no limbo foliar de numerosas pústulas pequenas, elípticas, a princípio recobertas pela cutícula da folha. Com o rompimento desta há exposições de massa pulverulenta, de cor amarela, constituída por uredosporos do fungo. Numa fase mais avançada da doença, a massa pulverulenta formada na pústula mostra-se de cor castanho-escura ou preta, devido à formação de teliósporos (Massola Jr. et al., 2005).
Com relação ao manejo da cultura, é importante evitar o plantio em solos compactados, de baixada, bem como adubações desequilibradas. A utilização de cultivares mais resistentes à doença é recomendada e o controle químico é efetivo, sendo utilizado com freqüência nas lavouras de alho.

Fungicidas do grupo dos triazóis são recomendados para o controle da ferrugem (Massola Jr. et al., 2005). Entre eles o tebuconazole é utilizado com bons resultados de controle da doença e seletividade à cultura (Sanchez et al., 2003; Andrei, 2005). Os produtos do grupo das estrobilurinas são de uso mais recente e mostram bons resultados de controle de diferentes doenças e culturas, especialmente quando em mistura pronta com triazóis, aumentam o espectro de controle e a ação residual do tra- 
Tabela 1. Severidade (\%) de Puccinia porri e controle (\%) por fungicidas aplicados seqüencialmente em três épocas na cultura do alho (Allium sativum L.) (Severity (\%) of Puccinia porri and control (\%) through fungicides applied sequentially in three times in the garlic culture). Ponta Grossa, UEPG, 2003.

\begin{tabular}{|c|c|c|c|c|c|c|c|}
\hline \multirow{2}{*}{ Nome comercial } & \multirow{2}{*}{$\begin{array}{l}\text { Dose }^{1} \\
\left(\text { g ha-1) }^{-1}\right)\end{array}$} & \multicolumn{2}{|c|}{$11 \mathrm{DAA}^{2}$} & \multicolumn{2}{|c|}{18 DAA } & \multicolumn{2}{|c|}{27 DAA } \\
\hline & & Sever. & $\% \mathrm{C}^{3}$ & Sever. & $\% \mathrm{C}$ & Sever. & $\% \mathrm{C}$ \\
\hline 1. Testemunha & 0 & $14,8 \mathrm{a}$ & - & $25,0 \mathrm{a}$ & - & 25,0 a & - \\
\hline 2. Tebuconazole ${ }^{4}$ & 200 & $2,5 \mathrm{e}$ & 83,4 & $4,5 \mathrm{e}$ & 82,0 & $9,4 \mathrm{c}$ & 62,5 \\
\hline 3. Trifloxystrobin e tebuconazole ${ }^{5}$ & 50 e 100 & $5,7 \mathrm{c}$ & 61,7 & $10,0 \mathrm{c}$ & 60,0 & $15,0 \mathrm{~b}$ & 40,0 \\
\hline 4. Trifloxystrobin e tebuconazole ${ }^{5}$ & 75 e 150 & $3,9 \mathrm{~d}$ & 74,0 & $5,3 \mathrm{de}$ & 79,0 & $8,5 \mathrm{~cd}$ & 66,0 \\
\hline 5. Trifloxystrobin e tebuconazole ${ }^{6}$ & 50 e 100 & $8,1 \mathrm{~b}$ & 45,1 & $4,5 \mathrm{~b}$ & 42,0 & $12,5 b$ & 50,0 \\
\hline 6. Trifloxystrobin e tebuconazole ${ }^{6}$ & 75 e 150 & $4,3 \mathrm{~d}$ & 71,3 & $6,5 \mathrm{e}$ & 74,0 & $6,3 d$ & 75,0 \\
\hline C.V. $(\%)$ & - & 4,3 & - & 4,8 & - & 5,3 & - \\
\hline
\end{tabular}

Médias seguidas de letras iguais na coluna, não diferem pelo teste de Tukey $(\mathrm{p}<5 \%)$; ${ }^{1}$ Dose em gramas por hectare; ${ }^{2} \mathrm{DAA}=$ dias após a primeira aplicação dos fungicidas; ${ }^{3 \%} \mathrm{C}=$ porcentagem de controle; ${ }^{4}$ Tratamento utilizado como padrão; ${ }^{5}$ Formulação SC; ${ }^{6}$ Formulação WG (Means followed by the same letter in the column, did not differ through the Tukey test $(\mathrm{p}<5 \%)$; ${ }^{1}$ Rate in gram for hectare; ${ }^{2} \mathrm{DAA}=\mathrm{days}$ after the first application of the fungicides; ${ }^{3 \%} \mathrm{C}=$ control percentage; ${ }^{4}$ Treatmento used as standard; ${ }^{5} \mathrm{SC}$ Formularization; ${ }^{6} \mathrm{WG}$ Formularization).

tamento (Picinini \& Prestes, 1994; Picinini \& Fernandes, 1998). Para o controle da ferrugem do alho ainda não há relatos na literatura a respeito do uso combinado de trifloxystrobin com tebuconazole, mas essa mistura mostra resultados eficientes no controle de ferrugem em cevada (Rizzi et al., 2006), ferrugem em feijão (Modesto et al., 2005) e brusone no arroz (Lobo, 2004) sem causar efeitos fitotóxicos nas plantas.

O presente trabalho teve como objetivo avaliar se há a vantagem da adição do fungicida trifloxystrobin ao tebuconazole no controle de ferrugem na cultura do alho.

\section{MATERIAL E MÉTODOS}

$\mathrm{O}$ experimento foi instalado na $\mathrm{Fa}-$ zenda Escola "Capão da Onça" da UEPG, Município de Ponta Grossa, PR, em um Cambissolo distrófico de textura argilosa, no ano 2003. O delineamento experimental utilizado foi de blocos ao acaso com seis tratamentos e quatro repetições. As parcelas apresentaram área total de $6,0 \mathrm{~m}^{2}(5,0 \times 1,2 \mathrm{~m})$ e área útil de $4,0 \mathrm{~m}^{2}(4,0 \times 1,0 \mathrm{~m})$.

O plantio do alho, cultivar Caçador, foi realizado manualmente em 15/07/03, em fileiras espaçadas de $0,25 \mathrm{~m}$ e 0,10 $\mathrm{m}$ entre plantas. A adubação consistiu da aplicação de $1.000 \mathrm{~kg} \mathrm{ha}^{-1}$ de adubo de fórmula comercial 04-20-20 no plantio e $50 \mathrm{~kg} \mathrm{ha}^{-1}$ de nitrogênio em cobertura na forma de uréia. $\mathrm{O}$ controle de plantas daninhas foi realizado manualmente na área do experimento.
Os tratamentos constaram de tebuconazole na dose de $200 \mathrm{~g} \mathrm{ha}^{-1}(1,00$ $\mathrm{L} \mathrm{ha}^{-1}$ de Folicur $200 \mathrm{CE}$, concentrado emulsionável); e das misturas formuladas de trifloxystrobin e tebuconazole nas doses de 50 e 100 e 75 e $150 \mathrm{~g} \mathrm{ha}^{-1}(0,50$ e $0,75 \mathrm{~L} \mathrm{ha}^{-1}$ de Nativo SC, suspensão concentrada); trifloxystrobin e tebuconazole nas doses $50 \& 100$ e 75 e $150 \mathrm{~g} \mathrm{ha}^{-1}\left(0,20\right.$ e $0,30 \mathrm{~kg} \mathrm{ha}^{-1}$ de Nativo WG, granulado dispersível) e testemunha. Os fungicidas foram aplicados através de pulverizador costal, à pressão constante de $35 \mathrm{lb} \mathrm{pol}^{-2}$, mantida por $\mathrm{CO}_{2}$ comprimido, com pontas de jato plano "leque" XR 110.02 e volume de calda de $500 \mathrm{~L} \mathrm{ha}^{-1}$. Foram realizadas três aplicações dos tratamentos, aos 85, 96 e 103 dias após a emergência do alho.

Foi avaliada a severidade de Puccinia porri, determinando-se a severidade, utilizando a escala diagramática de Azevedo (1997). Foram avaliadas as folhas de 10 plantas por parcela aos 85, 96 e 103 dias após a emergência das plantas de alho, correspondente a 11, 18 e 27 dias após a primeira aplicação dos tratamentos.

Os dados foram submetidos à análise da variância (teste F) e as diferenças entre as médias, quando significativas, comparadas pelo teste de Tukey no nível de $5 \%$ de probabilidade. Para as avaliações de severidade os dados foram transformados para arc sen $\sqrt{x+0,5 / 100}$.

\section{RESULTADOS E DISCUSSÃO}

O clima no decorrer do experimento caracterizou-se por temperaturas amenas e alta umidade relativa do ar, o que é favorável ao desenvolvimento da ferrugem (Massola Jr. et al., 2005). Assim a severidade da doença foi bastante alta, como pode ser notado pelos resultados da testemunha (Tabela 1). A severidade foi máxima pela escala utilizada já aos 18 dias após a aplicação (DAA) dos tratamentos. Em razão dessa alta severidade, mesmo para o tebuconazole, que é recomendado para o controle da doença (Souza et al., 2003; Sanchez et $a l ., 2003)$, ocorreu redução na porcentagem de controle nas avaliações realizadas aos 18 e 27 DAA. Em relação ao trifloxystrobin e tebuconazole (SC) e a trifloxystrobin e tebuconazole (WG) nas maiores doses (75 e $150 \mathrm{~g} \mathrm{ha}^{-1}$ e 75 e $150 \mathrm{~g} \mathrm{ha}^{-1}$ respectivamente), os resultados foram similares e o controle não diferiu do tebuconazole na avaliação aos 18 DAA, com controle acima de $74 \%$. Estes mesmos produtos e formulações nas menores doses foram estatisticamente inferiores às maiores doses, aos 18 DAA, indicando o aumento da eficiência do controle da ferrugem com o aumento das doses dos produtos.

Em trabalho realizado por Sanchez et al. (2003), foi observado que o tebuconazole na dose de $200 \mathrm{~g}^{\text {ia }} \mathrm{ha}^{-1}$ apresentou excelente eficácia de controle da ferrugem na cultura do alho, o que não aconteceu no presente trabalho pela alta severidade em que a doença ocorreu.

Embora houvessem diferenças no controle entre os fungicidas, essas não foram suficientes para se refletir na pro- 
Tabela 2. Produção da cultura do alho (Allium sativum L.) em função de fungicidas aplicados visando o controle de Puccinia porri (yield of the garlic culture depending on the applied fungicides to control the Puccinia porri). Ponta Grossa, UEPG, 2003.

\begin{tabular}{|c|c|c|}
\hline Nome comercial & $\operatorname{Dose}^{1}\left(g_{\text {ha-1 }}\right)$ & Produção $\left(\mathrm{g} \mathrm{m}^{-2}\right)$ \\
\hline 1. Testemunha & 0 & $875,0 \quad b$ \\
\hline 2. Tebuconazole ${ }^{2}$ & 200 & $1.300,0 \mathrm{a}$ \\
\hline 3. Trifloxystrobin e tebuconazole ${ }^{3}$ & 50 e 100 & $1.047,5 \mathrm{ab}$ \\
\hline 4. Trifloxystrobin e tebuconazole ${ }^{3}$ & 75 e 150 & $997,5 a b$ \\
\hline 5. Trifloxystrobin e tebuconazole ${ }^{4}$ & 50 e 100 & $1.012,5 \mathrm{ab}$ \\
\hline 6. Trifloxystrobin e tebuconazole ${ }^{4}$ & 75 e 150 & $965,0 \mathrm{ab}$ \\
\hline C.V. $(\%)$ & - & $6,8 \%$ \\
\hline
\end{tabular}

Médias seguidas de letras iguais na coluna, não diferem pelo teste de Tukey $(\mathrm{p}<5 \%)$; ${ }^{1}$ Dose gramas por hectare; ${ }^{2}$ Tratamento utilizado como padrão; ${ }^{3}$ Formulação SC; ${ }^{4}$ Formulação WG (means followed by the same letter in the column, did not differ through the Tukey test $(\mathrm{p}<5 \%) ;{ }^{1}$ Rate in gram for hectare; ${ }^{2}$ Treatment used as standard; ${ }^{3} \mathrm{SC}$ Formulation; ${ }^{4} \mathrm{WG}$ Formulation).

dução, que foi similar entre esses (Tabela 2). O único tratamento que proporcionou produção superior ao da testemunha foi tebuconazole. Não foram observadas diferenças substanciais no controle entre as formulações da mistura de trifloxystrobin e tebuconazole, sendo ambos adequados no controle da ferrugem.

Não foram observadas alterações nas plantas de alho que pudessem ser atribuídas aos fungicidas utilizados. Nas condições em que foi desenvolvido o experimento, de alta severidade de ferrugem, pode-se concluir que a mistura de trifloxystrobin e tebuconazole na dose de 75 e $150 \mathrm{~g} \mathrm{ha}^{-1}$ das formulações SC e WG é adequada ao controle da ferrugem do alho pela similaridade de controle ao tebuconazole, que é recomendado para o controle da doença, e por não causar danos visíveis na cultura. As misturas formuladas de trifloxystrobin e tebuconazole contêm a mesma quantidade de tebuconazole do Folicur 200 $\mathrm{CE}$ e preço superior em 5 a $15 \%$, sendo vantajosa a utilização da mistura por conter o trifloxystrobin que, mesmo não tendo sido observada vantagem no controle da ferrugem, é um fungicida que controla outras doenças, especialmente quando aplicado preventivamente (Venâncio et al., 1999). Ainda, o uso de produtos de grupos químicos diferentes em mistura formulada constitui importante estratégia para evitar a resistência dos patógenos a fungicidas (Reis et al., 2007). Das misturas formuladas, a empresa fabricante optou por registrar a formulação SC, que permite o uso de menores quantidades de tebuconazole na mistura em comparação ao produto isolado. Isso confere ao agricultor a possibilidade de escolha dos produtos em função das doenças que ocorrem, com pequeno acréscimo de preço.

\section{REFERÊNCIAS}

ANDREI, E. 2005. Compêndio de Defensivos Agrícolas. 7 ed. São Paulo: Organização Andrei Editora Ltda. 1141p.

AZEVEDO LAS. 1997. Manual de quantificação de doenças de plantas. Ed. do autor. 114p.

LOBO VLS. 2004. Tratamento Químico para o Controle da Brusone na Panícula. EMBRAPA, Santo Antônio de Goiás. Comunicado Técnico, $\mathrm{n}^{\circ} 78$.
MASSOLA JUNIOR NS; JESUS JUNIOR WC; KIMATI H. 2005. Doenças do alho e da cebola. In: KIMATI $\mathrm{H}$; AMORIN L; REZENDE JAM; BERGAMIN FILHO A; CAMARGO LEA. 2005. Manual de Fitopatologia. São Paulo: Agronômica Ceres. p. 56.

MODESTO JC; FENILLE RC; HABERMANN G. 2005. Efeito de fungicidas no controle da ferrugem do feijoeiro causada por Uromyces appendiculatus em condições de campo. Arquivos do Instituto Biológico 72: 245-248.

PICININI EC; FERNANDES JMC. 1998. Eficácia do fungicida propiconazole e de sua mistura com cyproconazole e difenoconazole no controle de doenças da parte aérea na cultura do trigo. Fitopatologia Brasileira 23: 270. (Suplemento).

PICININI EC; PRESTES AM. 1994. Avaliação de fungicidas no controle de doenças de trigo. In: REUNIÃO NACIONAL DE PESQUISA DE TRIGO, 13. 1994. Cruz Alta. Resultados de pesquisa, Passo Fundo, Embrapa/CNPT. p.229-231.

REIS EM; REIS AC; FORCELINI CA. 2007. Manual de fungicidas. Guia para o controle químico de doenças de plantas. Passo Fundo: Editora Universidade de Passo Fundo. 153 p.

RIZZI FP; FEKSA HR; DUHATSCEK B. 2006. Manejo do complexo de doenças na cultivar de cevada BRS 195, Entre Rios - Guarapuava/ PR. Fitopatologia Brasileira 31: 376. (Resumos)

SANCHEZ W; ABRAMSSON VR; FELIPPE JM. 2003. Controle da ferrugem (Puccinia allii) do alho (Allium sativum) com o fungicida Pyraclostrobin + Metiram. Fitopatologia Brasileira 28: 303. (Resumos)

SOUZA TA; VENANCIO WS; RODRIGUES MAT; BEGLIOMINI E. 2003. Avaliação da eficiência de fungicidas no controle da mancha púrpura (Alternaria porri) e da ferrugem (Puccinia allii) na cultura do alho (Allium sativum L.). Fitopatologia Brasileira 28: 335. (Resumos).

VENANCIO WS; ZAGONEL J; FURTADO EL; SOUZA NL. 1999. Novos fungicidas I Produtos Naturais e Derivados Sintéticos: Estrobrlulinas e Fenilpirroles. In: Wilmar Corio da Luz; Jose Mauricio Cunha Fernandes; Adriano Moraes Prestes; Edson Clodoveu Picinini. (Org.). Revisão Anual de Patologia de Plantas. 1 ed. Passo Fundo: Revisão Anual de Patologia de Plantas, v. 7, p. 103-155. 